\title{
Seguridad e Higiene en las empresas de la Zona Norte del Estado de Guerrero
}

\section{Safety and Hygiene in companies in the Northern Zone of the State of Guerrero}

\author{
MANUEL-REGINO, Fernando †*, MARCHÁN-LÁZARO, Rosalía, BÁRCENAS-NAVA, Ma. de los \\ Ángeles, TRUJILLO-BELTRÁN, Elisa y MONTES-CARMONA, Sandra Guadalupe
}

Tecnológico Nacional de México (TecNM), Campus Instituto Tecnológico de Iguala. Carretera Nacional Iguala-Taxco esquina Periférico Norte S/N, Colonia Adolfo López Mateos INFONAVIT, C.P. 40030, Iguala de la Independencia, Guerrero, Teléfono 01 (733) 1011074.

ID $1^{\text {er }}$ Autor: Fernando, Manuel-Regino / ORC ID: 0000-0002-0457-9166, CVU CONACYT ID: 540594

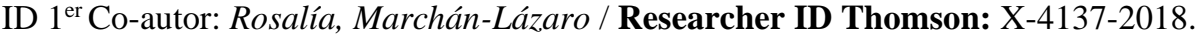

ID $2^{\text {do }}$ Co-autor: Ma. De los Ángeles, Bárcenas-Nava / ORC ID: 0000-0003-2693-9473, Researcher ID Thomson: X4133-2018, Researcher ID Thomson: X-4137-2018, CVU CONACYT ID: 550869

ID $3^{\text {er }}$ Co-autor: Elisa, Trujillo-Beltrán

ID $4^{\text {to }}$ Co-autor: Sandra Guadalupe, Montes-Carmona

DOI: $10.35429 /$ JLA.2019.10.3.21.26

Recibido 10 de Octubre, 2019; Aceptado 23 de Diciembre, 2019

\section{Resumen}

Toda Persona Física o Moral que desarrolla una actividad empresarial, de Servicios o Industrial está obligada a cumplir disposiciones legales en materia de Seguridad e Higiene con sus trabajadores, que garantice en todo momento la salud, bienestar y la conservación de la vida humana, a fin de prevenir accidentes, riegos laborales y enfermedades profesionales que puedan producirse durante y después de su jornada laboral. El objetivo de la presente investigación es hacer un diagnóstico de las empresas de la Zona Norte del Estado de Guerrero, que al contratar trabajadores cumplen con las leyes laborales y de seguridad social en sus organizaciones (Oficina, talleres, área de producción, maquinas, con el fin de evitar el pago de multas y sanciones por parte de la secretaría del Trabajo y Previsión Social y de capitales constitutivos por parte del Seguro Social. Damos las gracias al Tecnológico Nacional de México campus Instituto Tecnológico de Iguala, por el apoyo económico y las facilidades para la culminación de esta Investigación.

Seguridad, Higiene, leyes laborales, accidentes de trabajo

\begin{abstract}
Every Physical or Moral Person that develops a business, Services or Industrial activity is obliged to comply with legal provisions in matters of Safety and Hygiene with its workers, which guarantees at all times the health, well-being and preservation of human life, in order to prevent accidents, occupational hazards and occupational diseases that may occur during and after your workday. The objective of the present investigation is to make a diagnosis of the companies of the North Zone of the State of Guerrero, that when hiring workers comply with the labor and social security laws in their organizations (Office, workshops, production area, machines, with In order to avoid the payment of fines and sanctions by the Ministry of Labor and Social Welfare and constituent capital by Social Security, we thank the Technological National of Mexico campus, Instituto Tecnológico de Iguala, for the financial support and facilities for the completion of this Research.
\end{abstract}

Safety, Hygiene, labor laws, accidents at work

Citación: MANUEL-REGINO, Fernando, MARCHÁN-LÁZARO, Rosalía, BÁRCENAS-NAVA, Ma. de los Ángeles, TRUJILLO-BELTRÁN, Elisa y MONTES-CARMONA, Sandra Guadalupe. Seguridad e Higiene en las empresas de la Zona Norte del Estado de Guerrero. Revista de Aplicaciones del Derecho. 2019. 3-10: 21-26.

\footnotetext{
*Correspondencia al Autor (Correo Electrónico: cpcfermaregis@ live.com.mx)

$\dagger$ Investigador contribuyendo como primer autor.
} 


\section{Introducción}

La presente investigación se realizó en el Estado de Guerrero, el cual se divide en 7 regiones que concentran los 81 municipios del estado, éstas son: Acapulco, Centro, Zona Norte, Tierra Caliente, Costa Chica, Costa Grande y La Montaña, la Ciudad de Iguala de la Independencia y el Instituto Tecnológico de Igual, se encuentra ubicado en la Zona norte del Estado de Guerrero, donde se gesta la presente investigación, como se muestra en la Figura 1

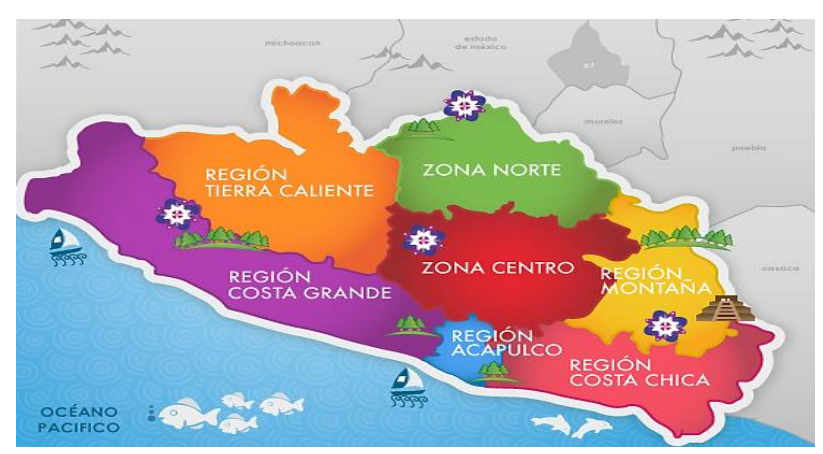

Figura 1 Zonas del Estado de Guerrero

Fuente: Portal Oficial guerrero.gob.mx

Para dar inicio a nuestro tema, podemos señalamos que toda Persona Física o Moral que desarrolla una actividad empresarial, Comercial, de Servicios o Industrial en la Ciudad de Iguala de la Independencia, está obligada a cumplir disposiciones en materia de Seguridad e Higiene con sus trabajadores, a fin de garantizar la vida humana, es decir que no lo vean como una opción sino como una obligación que emana principalmente de nuestra constitución Política de los Estados Unidos Mexicanos, las leyes laborales y de seguridad social.

Según la American Industrial Hygienist Assocciation (A.I.H.A.), la Higiene Industrial es la "Ciencia y arte dedicados al reconocimiento, evaluación y control de aquellos factores ambientales o tensiones emanados o provocados por el lugar de trabajo y que pueden ocasionar enfermedades, destruir la salud y el bienestar o crear algún malestar significativo entre los trabajadores o los ciudadanos de una comunidad". La Higiene Industrial también podría definirse como la disciplina dentro del marco de la prevención de riesgos laborales que se encarga del conjunto de procedimientos destinado a controlar los factores ambientales que pueden afectar a la salud de los trabajadores en el ámbito laboral.
Así, la higiene industrial debe ser capaz de identificar, evaluar y eliminar los agentes que pueden causar efectos negativos sobre los trabajadores, ya sean estos agentes físicos, químicos o biológicos. (Frades \& Godoy, 2013) La seguridad se define como: la confianza, situación en la que está ha cubierto de un riesgo. $\mathrm{Su}$ aplicación es a los dispositivos destinados a evitar accidentes. La Seguridad Social se define como el conjunto de leyes y de los organismos que las aplican, que tienen por objeto proteger a la sociedad contra determinados riesgos. La higiene laboral se refiere a un conjunto de normas y procedimientos que buscan proteger la integridad física y mental de los trabajadores, vigilar los riesgos de salud inherentes a las tareas del puesto y al ambiente físico donde las realiza. Tienen carácter preventivo ya que tienen por objeto la salud y comodidad del trabajador, evitando que se enferme y se ausente provisional o definitivamente del trabajo. (Psicologia y empresa, 2009)

Desde el nacimiento de las industrias el hombre se vio expuesto a diferentes riesgos, accidentes o enfermedades durante las jornadas de trabajo. Los trabajadores en México, así como los de otros países estuvieron expuestos a circunstancias de riesgo laboral y del abandono por parte de los empresarios, quienes no tenían interés en la seguridad de los trabajadores, estas situaciones los llevaron a tomar la iniciativa para llevar a cabo acciones que disminuyeran los riesgos a los que estaban expuestos.

En general, en la mayoría de los problemas de las industrias, se suele trabajar con un único objetivo, como puede ser maximizar beneficios o minimizar gastos. Sin embargo, como todos sabemos, en las empresas los objetivos son múltiples y a veces, incluso excluyentes, es decir, el cumplimiento de un objetivo se hace a costa de incumplir otros, pero en el caso de seguridad e higiene esto no debe descuidarse, teniendo en cuenta que las enfermedades y riesgos laborales deberían ser llevados a cero o eliminarlos, a través de una mejora continua.

La Seguridad e higiene comienza con el desarrollo de la Revolución Industrial y fue en Inglaterra en el siglo XVIII los primeros datos conocidos (Hernández, 2005), en Inglaterra nacieron las primeras leyes en el año de 1855 en América se han ido dando posteriormente. 
Al hablar de higiene nos referimos a la prevención de enfermedades o accidentes laborales que pueden ser ocasionadas por factores físicos, biológicos y químicos. La higiene tiene como propósito identificar, medir, evaluar y controlar estos factores que pueden afectar a los trabajadores en su ambiente de trabajo. Por lo tanto el patrón se ve comprometido a proporcionar todos los medios necesarios tales como la capacitación y adiestramiento en relación a seguridad e higiene (Mateo, 2007).

En México en el año de 1910 los trabajadores mexicanos contaban con el amparo de solo dos leyes a nivel local. La primera ley fue designada como José Vicente Villada en el año de 1904 y sus disposiciones solo estaban para el Estado de México, la segunda dos años después fue nombrada Bernardo Reyes y su aplicación solo tenía efectos en el estado de Nuevo León, en estas dos leyes quedo estipulado al patrón como el responsable de los accidentes laborales y por ello debería pagar una compensación por los percances ocurridos durante la jornada laboral. (Hernández A. , 2005). La Reglamentación legal que establece nuestro país para delimitar las condiciones laborales que debe tener una empresa en aspectos de seguridad e higiene están sujetos a las siguientes Leyes: 1. Constitución Política de los Estados Unidos Mexicanos, 2. Ley Federal del Trabajo, 3. Reglamento de Seguridad e Higiene, 4. Ley del Seguro Social y 5. Normas Oficiales Mexicanas.

Dentro del marco legal de nuestro tema analizaremos como primer punto, nuestra Constitución Política de los Estados Unidos Mexicanos, la cual señala en su artículo 123 apartado A, primer párrafo, precisa lo siguiente: "Toda persona tiene derecho al trabajo digno y socialmente útil; al efecto, se promoverán la creación de empleos y la organización social de trabajo, conforme a la ley”. (Diputados G. , 2019), Así mismo, en su fracción XIV, establece que "Los empresarios serán responsables de los accidentes del trabajo y de las enfermedades profesionales de los trabajadores, sufridas con motivo o en ejercicio de la profesión o trabajo que ejecuten; por lo tanto, los patronos deberán pagar la indemnización correspondiente, según que haya traído como consecuencia la muerte o simplemente incapacidad temporal o permanente para trabajar, de acuerdo con lo que las leyes determinen.
Esta responsabilidad subsistirá aún en el caso de que el patrono contrate el trabajo por un intermediario". De igual forma establece este mismo artículo en su fracción XV, lo siguiente, “ El patrón estará obligado a observar, de acuerdo con la naturaleza de su negociación, los preceptos legales sobre higiene y seguridad en las instalaciones de su establecimiento, y a adoptar las medidas adecuadas para prevenir accidentes en el uso de las máquinas, instrumentos y materiales de trabajo, así como a organizar de tal manera éste, que resulte la mayor garantía para la salud y la vida de los trabajadores, y del producto de la concepción, cuando se trate de mujeres embarazadas. Las leyes contendrán, al efecto, las sanciones procedentes en cada caso". Así mismo en la fracción XXI, establece que será competencia exclusiva de las autoridades federales la aplicación de las disposiciones de trabajo en los asuntos relativos a las "obligaciones de los patrones en materia de seguridad e higiene en los centros de trabajo, para lo cual las autoridades federales contarán con el auxilio de las Estatales, cuando se trate de ramas o actividades de jurisdicción local, en los términos de la ley reglamentaria correspondiente".

La Ley Federal del Trabajo, (Diputados g. , 2019), prevé las causas físicas y mecánicas de los accidentes, su aplicación en México ha tenido poco efecto debido a su impopularidad y la dificultad para hacerla cumplir. Sin embargo, las leyes que gravan a los patrones con los costos de los accidentes, han tenido efectos de mayor alcance, obligándolos a buscar y corregir las condiciones negativas.

El Mantener las áreas de trabajo seguras e higiénicas también es responsabilidad del trabajador cuando se den los siguientes supuestos de conformidad con el artículo 47 de esta ley la cual establece, son causas de rescisión de la relación de trabajo, sin responsabilidad para el patrón: VII. Comprometer el trabajador, por su imprudencia o descuido inexcusable, la seguridad del establecimiento o de las personas que se encuentren en él; XII. Negarse el trabajador a adoptar las medidas preventivas o a seguir los procedimientos indicados para evitar accidentes o enfermedades. 
De igual forma queda prohibido a los trabajadores de acuerdo al artículo 135, Ejecutar cualquier acto que pueda poner en peligro su propia seguridad, la de sus compañeros de trabajo o la de terceras personas, así como la de los establecimientos o lugares en que el trabajo se desempeñe. Para su mejor entendimiento la Ley Federal del Trabajo, para su mejor entendimiento, da a conocer los siguientes conceptos: Riesgos de trabajos. Son los accidentes y enfermedades a que están expuestos los trabajadores en ejercicio o con motivo del trabajo. Accidente de trabajo, es toda lesión orgánica o perturbación funcional, inmediata o posterior, o la muerte, producida repentinamente en ejercicio, o con motivo del trabajo, cualesquiera que sean el lugar y el tiempo en que se preste. Enfermedad de trabajo, es todo estado patológico derivado de la acción continuada de una causa que tenga su origen o motivo en el trabajo o en el medio en que el trabajador se vea obligado a prestar sus servicios. Incapacidad temporal, es la pérdida de facultades o aptitudes que imposibilita parcial o totalmente a una persona para desempeñar su trabajo por algún tiempo.

La Ley del Seguro Social, tiene a su cargo la protección del trabajador y su familia contra riesgos y enfermedades a que estén sujetos los trabajadores como motivo de su trabajo. El objetivo principal de la Ley del Seguro Social es garantizar el derecho a la salud, la asistencia médica, la protección de los medios de subsistencia y los servicios sociales necesarios para el bienestar individual y colectivo, así como el otorgamiento de una pensión que, en su caso y previo cumplimiento de los requisitos legales, será garantizada por el Estado, (Cámara de Diputados, 2019) Los riesgos de trabajo pueden producir: I. Incapacidad temporal, II.- Incapacidad permanente parcial, III. Incapacidad permanente total, y IV. Muerte. Señalado en su Artículo 55.

Las Normas Oficiales Mexicanas que emite la Secretaria del Trabajo y Previsión Social (STPS) de acuerdo al giro o actividad que realiza cada empresa. En el presente, se encuentran vigentes 41 normas oficiales mexicanas en materia de seguridad y salud en el trabajo. Dichas normas se agrupan en cinco categorías: de seguridad, salud, organización, específicas y de producto. Su aplicación es obligatoria en todo el territorio nacional. (STPS, 2018).
A continuación se enumerar únicamente como ejemplo las normas de seguridad $y$ organización, como lo señala las tablas 1 y 2 :

\begin{tabular}{|c|c|}
\hline Número & Título de la norma \\
\hline NOM-010-STPS-1999 & $\begin{array}{l}\text { Contaminantes por sustancias } \\
\text { químicas }\end{array}$ \\
\hline NOM-010-STPS-2014 & $\begin{array}{ll}\text { Agentes } & \text { químicos } \\
\text { contaminantes } & \text { del ambiente } \\
\text { laboral } & \\
\end{array}$ \\
\hline NOM-011-STPS-2001 & Ruido \\
\hline NOM-012-STPS-2012 & Radiaciones ionizantes \\
\hline NOM-015-STPS-2001 & $\begin{array}{l}\text { Condiciones térmicas } \\
\text { elevadas o abatidas }\end{array}$ \\
\hline NOM-025-STPS-2008 & Iluminación \\
\hline
\end{tabular}

Tabla 1 Normas Oficiales Mexicanas de Salud Fuente: elaboración propia

\begin{tabular}{|l|l|l|}
\hline \multicolumn{1}{|c}{ Número } & \multicolumn{1}{c|}{ Título de la norma } \\
\hline NOM-017-STPS-2008 & $\begin{array}{l}\text { Equipo de protección } \\
\text { personal }\end{array}$ \\
\hline NOM-018-STPS-2000 & $\begin{array}{l}\text { Identificación de peligros y } \\
\text { riesgos por sustancias } \\
\text { químicas }\end{array}$ \\
\hline NOM-019-STPS-2011 & $\begin{array}{l}\text { Comisiones de seguridad e } \\
\text { higiene }\end{array}$ \\
\hline NOM-026-STPS-2008 & $\begin{array}{l}\text { Colores y señales de } \\
\text { seguridad }\end{array}$ \\
\hline NOM-030-STPS-2009 & $\begin{array}{l}\text { Servicios preventivos de } \\
\text { seguridad y salud }\end{array}$ \\
\hline
\end{tabular}

Tabla 2 Normas Oficiales Mexicanas de organización Fuente: elaboración propia

\section{Objetivos}

Identificar a las empresas que cumplen con las disposiciones legales, Constitucionales, Laborales y de Seguridad Social en materia de seguridad e higiene, en la Ciudad de Iguala de la Independencia, Guerrero.

\section{Metodología a desarrollar}

La presente investigación será Mixta, tomando en consideración que se partirá con una investigación documental tomando como base las disposiciones laborales y de seguridad social contenidas en la Constitución Política de los Estados Unidos Mexicanos, a Ley Federal del Trabajo, La ley del Seguro Social, las disposiciones y reglamentación de la Secretaria del Trabajo y Previsión Social, así como también las publicaciones en artículos indexados, memorias en extensos, Internet, en libros, Revistas y Tesis. Así mismo será una investigación aplicada en virtud de que se utilizara el instrumento de la entrevista a personas expertas en Seguridad e Higiene y se aplicara un cuestionario a los diferentes empresarios sujetos de estudio.

MANUEL-REGINO, Fernando, MARCHÁN-LÁZARO, Rosalía, BÁRCENAS-NAVA, Ma. de los Ángeles, TRUJILLO-BELTRÁN, Elisa y MONTES-CARMONA, Sandra Guadalupe. Seguridad e Higiene en las empresas de la Zona Norte del Estado de Guerrero. Revista de Aplicaciones del Derecho. 2019 


\section{Resultados}

Se llevó a cabo una sección de entrevistas con los titulares de la seguridad e higiene con autoridades Estatales de la Ciudad de Chilpancingo, Iguala de Independencia, así como con las autoridades del Seguro Social, de igual forma se aplicó un cuestionario a los empresarios de diferentes giros como son los hoteleros, restauranteros, constructores, fábricas de tabicón, y de la industria de la confección, dividido en dos partes, el primero de conocimiento de las leyes laborales y el segundo de aplicación de las disposiciones, como lo muestra el cuadro 3 , y que a continuación se muestra:

\begin{tabular}{|l|r|l|}
\hline \multicolumn{1}{|c|}{ Categorias } & \multicolumn{2}{|c|}{ Items } \\
\hline CATEGORÍA DE & 1 & Conoce normas \\
CONOCIMIENTO & 2 & Cuenta con reglamento \\
& 3 & Cuenta seguro \\
& 4 & Capacita a su personal \\
& 5 & Programa de \\
& 6 & mantenimiento \\
\hline CATEGORÍA DE & 7 & Cuenta con señalización \\
APLICACIÓN & 8 & Obligaciones autoridades \\
& 9 & Indemniza a sus \\
& 10 & trabajadores \\
& 11 & Revisiones periódicas \\
& 12 & Eanciona trabajadores \\
& 13 & Cuentan simulacros alarma \\
& 14 & Análisis de riesgo \\
& 15 & Ha sido sancionado por \\
\hline
\end{tabular}

Tabla 3 Estructura del cuestionario Fuente: elaboración propia

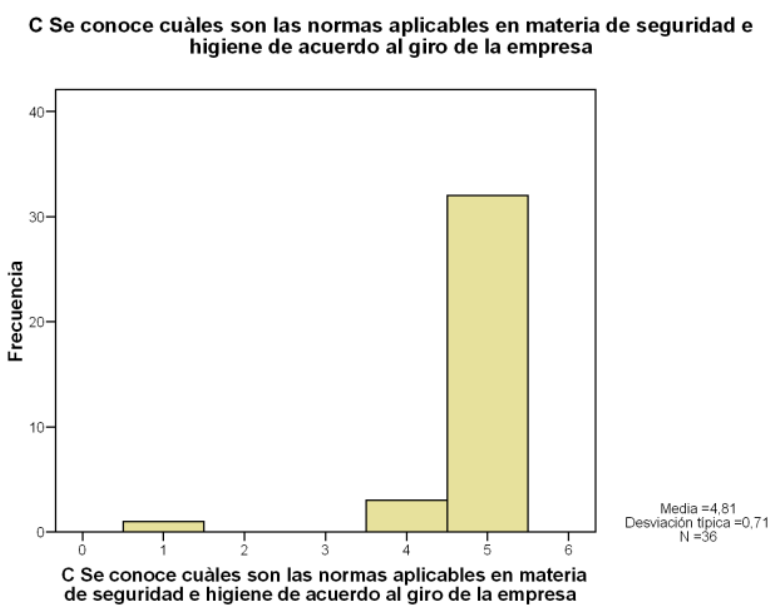

Gráfico 1 Conoce las normas de seguridad e higiene Fuente: elaboración propia

Como se muestra en el Gráfico 1, Los empresarios saben cuáles son las normas es en materia de seguridad e higiene de acuerdo al giro de la empresa, bajo la categoría 1 de aplicación.
La opción de respuesta que más se repitió fue 5 (siempre). Menos del cincuenta por cierto de los empresarios está por encima del valor 1 y el restante se sitúa por debajo de este valor (mediana).

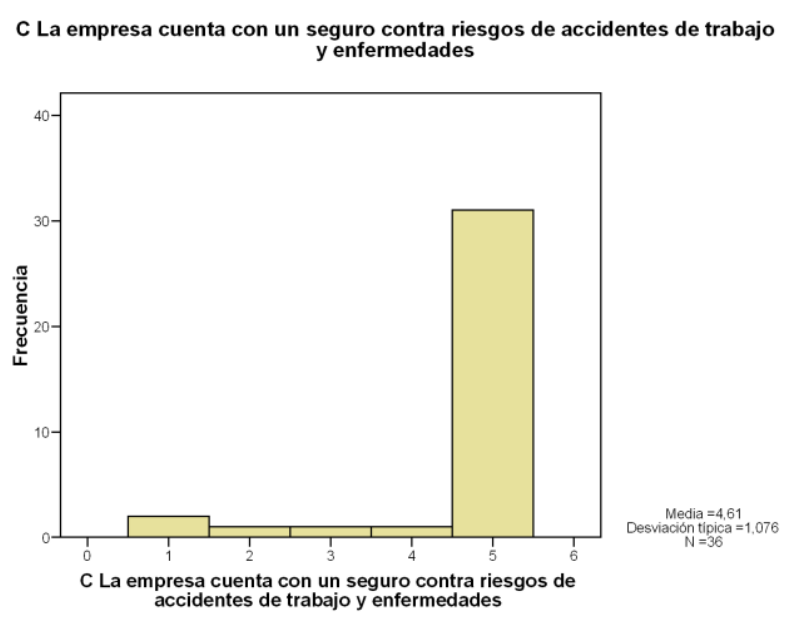

Gráfico 2 Cuenta con un reglamento

Fuente: elaboración propia

Como lo muestra el Gráfico 2, la respuesta que más se repitió fue 5 (si). Menos del cincuenta por cierto de los empresarios está por encima del valor 1 y el restante se sitúa por debajo de este valor (mediana). En promedio los empresarios se ubican en 4.81 (siempre). Ninguna persona califico de manera algunas veces, y casi nunca (no hay 2 y 3 ).

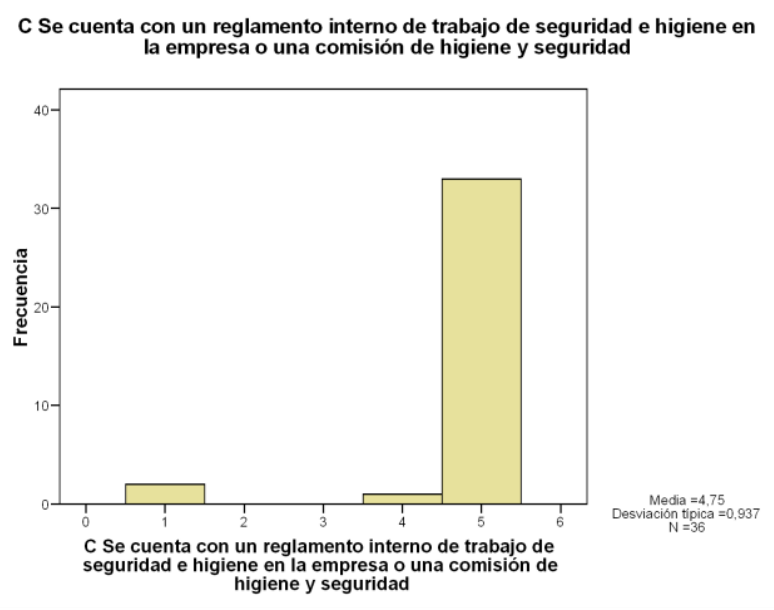

Gráfico 3 Cuenta con un reglamento

Fuente: elaboración propia

Como lo muestra el Gráfico 3, la respuesta que más se repitió fue 5 (si). Menos del cincuenta por cierto de los empresarios está por encima del valor 1 y el restante se sitúa por debajo de este valor (mediana). En promedio los empresarios se ubican en 4.81 (siempre). Ninguna persona califico de manera algunas veces, y casi nunca (no hay 2 y 3 ).

MANUEL-REGINO, Fernando, MARCHÁN-LÁZARO, Rosalía, BÁRCENAS-NAVA, Ma. de los Ángeles, TRUJILLO-BELTRÁN, Elisa y MONTES-CARMONA, Sandra Guadalupe. Seguridad e Higiene en las empresas de la Zona Norte del Estado de Guerrero. Revista de Aplicaciones empresas de la Zona Norte del Estado de Guerrero. Revista de Aplicaciones
del Derecho. 2019 


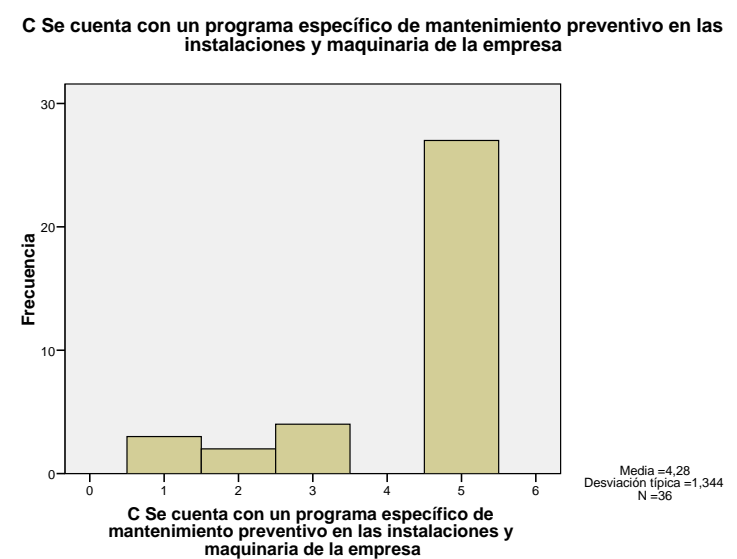

Gráfico 4 Se cuenta con un programa de mantenimiento preventivo

Fuente: elaboración propia

Como se puede observar, hace falta que los empresarios adopten una cultura laboral, que vean en sus trabajadores como aliados para incrementar sus ventas basado en la calidad de sus productos o servicios, desarrollando en sus organizaciones un clima seguro, higiénico evitando enfermedades y riesgo laboral, pero sobre todo evitar la pérdida de la vida humana.

\section{Agradecimiento}

Agradecemos al Tecnológico Nacional de México y a las autoridades académicas del Campus Instituto Tecnológico de Iguala, 'por todo el apoyo económico y las facilidades para el trabajo de campo.

\section{Contribución}

La presente investigación consideramos que el conocimiento de las leyes laborales que emanan de nuestra constitución no son una opción, sino una obligación de los empresarios, la contribución más importante de esta investigación sin lugar a duda, es la de crear una conciencia y cultura laboral que brinde a los trabajadores un clima de seguridad $\mathrm{y}$ armonía.

\section{Conclusiones}

La seguridad en el trabajo incluye todos aquellos actos, elementos o dispositivos que tiendan a preservar la integridad física de las personas y ponerlas en práctica a fin de evitar accidentes, y/o reducir la gravedad de los que se produzcan, la seguridad e higiene que están relacionadas con ciertos aspectos básicos como la ventilación, la humedad, temperatura, iluminación, los ruidos, la contaminación, etc.

Existen diferentes riesgos laborales, para ello es importante implementar las reglas de seguridad e higiene en el ambiente de trabajo. Podemos concluir, que la seguridad e higiene consiste en prevenir y controlar los riesgos originados durante una jornada de trabajo, garantizando el buen estado de salud de los trabajadores, a la par de preservar al medio ambiente y así contribuir a un desarrollo seguro y sostenible.

\section{Referencias}

Cámara de Diputados, M. (2019). Ley del Seguro Social. México: diputados.gob.mx.

Diputados, G. (2019). Constitución Política de los Estados Unidos Mexicanos. México: diputados.gob.mx.

Diputados, g. (2019). Ley Federal del Trabajo. México: diputados.gob.mx.

Frades, P., \& Godoy, R. (23 de Mayo de 2013). http://prevencionar.com. Obtenido de http://prevencionar.com/2013/05/23/gestion-dela-higiene-industrial/

Hernández, A. (2005). Seguridad e higiene industrial. México D.F.: Limusa.

Hernández, A. (2005). Seguridad e Higiene Industrial. México: limusa.

Mateo, P. (2007). GEstión de la Higiene Industrial en la empresa. Madrid España: Madrid FC.

Psicologia y empresa, c. (4 de Octubre de 2009). https://psicologiayempresa.com. Obtenido de https://psicologiayempresa.com/lahigiene-laboral.html

STPS, G. (2018). Comité Consultivo Nacional de Normalización de Seguridad y Salud en el Trabajo. México: gob.mx. 